Virginia Commonwealth University VCU Scholars Compass

2008

\title{
Unique magnetic coupling between Mn doped stannaspherenes Mn@Sn12
}

Anil K. Kandalam

Virginia Commonwealth University, akkandalam@vcu.edu

Gang Chen

Virginia Commonwealth University

Puru Jena

Virginia Commonwealth University, pjena@vcu.edu

Follow this and additional works at: http://scholarscompass.vcu.edu/phys_pubs

Part of the Physics Commons

Kandalam, A.K., Chen, G., Jena, P. Unique magnetic coupling between Mn doped stannaspherenes Mn@Sn12. Applied Physics Letters, 92, 143109 (2008). Copyright (C) 2008 AIP Publishing LLC.

\section{Downloaded from}

http://scholarscompass.vcu.edu/phys_pubs/19

This Article is brought to you for free and open access by the Dept. of Physics at VCU Scholars Compass. It has been accepted for inclusion in Physics Publications by an authorized administrator of VCU Scholars Compass. For more information, please contact libcompass@vcu.edu. 


\title{
Unique magnetic coupling between Mn doped stannaspherenes ${\mathrm{Mn} @ \mathrm{Sn}_{12}}$
}

\author{
Anil K. Kandalam, ${ }^{a}$ Gang Chen, and Puru Jena \\ Department of Physics, Virginia Commonwealth University, Richmond, Virginia 23284, USA
}

(Received 21 November 2007; accepted 21 February 2008; published online 10 April 2008)

\begin{abstract}
We report the density functional theory based study of the interaction between two Mn doped stannaspherenes $\left(\mathrm{Mn} @ \mathrm{Sn}_{12}\right)$. The calculated results show that $\mathrm{Mn} @ \mathrm{Sn}_{12}$ cluster is not only highly stable and carry a high magnetic moment, but these clusters retain their structural identity and form a stable dimer cluster. Most importantly, the magnetic coupling between the Mn@ $\mathrm{Sn}_{12}$ clusters depends on the relative orientation of the cages. In addition, $a b$ initio molecular dynamics calculations show that the dimer cluster is stable at room temperature. These results are expected to trigger further investigations on highly stable bimetallic magnetic cage complexes. (C) 2008 American Institute of Physics. [DOI: 10.1063/1.2896608]
\end{abstract}

The discovery of carbon fullerenes ${ }^{1,2}$ has stimulated considerable interest in the search of cage clusters involving not only group IV elements but also metallic systems. In particular, considerable amount of earlier work ${ }^{3-8}$ on $\mathrm{Si}$ and $\mathrm{Ge}$ failed to find neutral clusters of these elements to assume cage structures, although with suitable endohedral doping, clusters of these elements can be stabilized in cage form. Similarly, no neutral clusters of homonuclear metal atoms have been found to assume cage structures, although it was demonstrated $^{9}$ a long time ago that metal clusters can be stabilized in icosahedric cage structures by suitable endohedral doping. Recent discoveries ${ }^{10,11}$ of $\mathrm{Au}_{n}^{-}(n=16-18)$ and $\mathrm{Sn}_{12}^{2-}$ having cage structures have rekindled interest in metal fullerenes. Cui et al. have reported ${ }^{11}$ the discovery of stannaspherene, a highly stable $\mathrm{Sn}_{12}^{2-}$ cage cluster. This doubly charged $\mathrm{Sn}_{12}$ cage cluster, with icosahedral $\left(I_{h}\right)$ symmetry was found to be stable even without the aid of any central dopant atom. Part of the interest in stannaspherenes arises because these cage clusters have diameters large enough $(6.1 \AA)$ to accommodate a transition metal atom. ${ }^{11}$ In a recent experimental and theoretical studies, ${ }^{12} \mathrm{M} @ \mathrm{Sn}_{12}^{-} I_{h}$ cages $(M=\mathrm{Cu}, \mathrm{Ni}, \mathrm{Co}, \mathrm{Fe}, \mathrm{Cr}, \mathrm{V}, \mathrm{Ti}, \mathrm{Au}, \mathrm{Pt}$, and $\mathrm{Nb}$ ) were reported. Since transition metal atoms carry sizeable magnetic moments, it is possible to synthesize new magnetic materials with the doped metal fullerenes as building blocks. It should be emphasized that for the synthesis of new magnetic materials, it is not only necessary for these building blocks to carry a sizeable magnetic moment but also important to understand how they are coupled. No study has been carried out in this regard. In this communication, we show that Mn encapsulated $\mathrm{Sn}_{12}$ cluster is not only stable and carry a large magnetic moment but also their structure remains unaltered when interacted with each other even at room temperature. Equally important, the magnetic coupling between these clusters depends upon their relative orientation.

There are a number of reasons why $\mathrm{Mn} @ \mathrm{Sn}_{12}$ cluster is a candidate of choice: (1) among the $3 d$-transition metals, Mn predominantly occurs in divalent state due to its halffilled $3 d$ and filled $4 s$ shell and a significant energy gap separating the two orbitals. Thus, $\mathrm{Mn}$ doped $\mathrm{Sn}_{12}$ cluster can be viewed as $\mathrm{Mn}^{2+}: \mathrm{Sn}_{12}^{2-}$ cluster. Since, $\mathrm{Sn}_{12}^{2-}$ is a highly stable cluster, ${ }^{11}$ it is expected that the $\mathrm{Mn}$ doped $\mathrm{Sn}_{12}$ cluster

${ }^{a)}$ Electronic mail: akkandalam@vcu.edu. would also be very stable. (2) The special electronic structure of the Mn atom allows it to carry a magnetic moment of $5 \mu_{B}$ which remains intact when $\mathrm{Mn}$ atoms form clusters or compounds. (3) The magnetic coupling between the Mn atoms is very sensitive to their interatomic distance and environment. These raise some important questions: do these endohedral stannaspherene cages retain their structural integrity when they are brought near each other? What happens to the magnetic properties of such cages when they interact with each other? In order to answer these questions we have carried out extensive theoretical calculations.

All theoretical calculations were carried out using density functional theory with the Perdew-Wang $91^{13}$ (PW91) functional for the generalized gradient approximation for exchange and correlation potential. The geometries were optimized using the DMOL3 software. ${ }^{14}$ Semilocal pseudopotentials $^{15}$ for the core electrons, along with double numeric basis sets with polarization functions were used to describe Mn and Sn atoms. For the self-consistent field calculations, a convergence criterion for energy was set to $10^{-6}$ hartree, while density tolerance was set to $10^{-6} \mathrm{e} / \mathrm{bohr}^{3}$. In the geometry optimization procedure, the structural parameters of various isomers were fully optimized for all possible spin states without any symmetry restrictions. Accuracy of our calculated results was confirmed by carrying out independent calculations on the selected lower energy isomers using Vienna $a b$ initio simulation package (VASP). ${ }^{16,17}$ The spin-polarized calculations were performed within the framework of density functional theory with a plane wave basis set and projector-augmented-wave (PAW) potential, ${ }^{18,19}$ as implemented VASP. ${ }^{16,17}$ The PW91 generalized gradient approximation for exchange-correlation functional was used. The cut-off energy is $270 \mathrm{eV}$ for the plane wave basis set. A $26 \times 18 \times 18 \AA^{3}$ monoclinic supercell was used. The solution to the Kohn-Sham equation was calculated by an efficient matrix diagonalization technique based on a sequential bandby-band residual minimization method and a Pulay-type charge density mixing. ${ }^{17}$ Due to the large supercell, the Brillouin zone integration was carried out only at the $\Gamma$ point. In order to check the thermal stability of the ground state cluster, we have carried out molecular dynamics simulations. The constant temperature molecular dynamics simulations were performed at $300 \mathrm{~K}$ and lasted for $1 \mathrm{ps}$. The canonical ensemble was simulated by means of a Nosé thermostat ${ }^{20-22}$ as implemented in the VASP software package. 


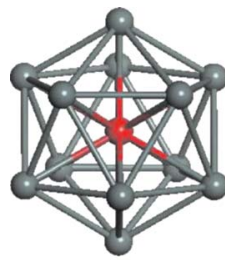

I $2 \mathrm{~s}+1=6$ $\Delta \mathrm{E}=\mathbf{0 . 0} \mathrm{eV}$

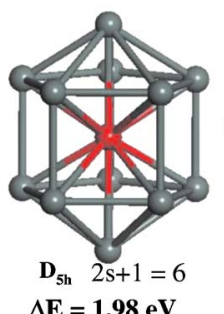

$\Delta \mathrm{E}=1.98 \mathrm{eV}$

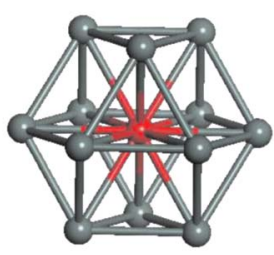

O. $2 \mathrm{~s}+1=6$

$\Delta \mathrm{E}=2.22 \mathrm{eV}$

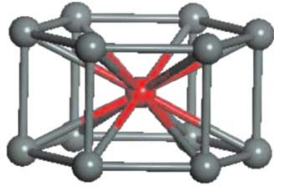

D $_{\text {6h }} 2 s+1=2$

$\Delta \mathrm{E}=\mathbf{2 . 2 0} \mathrm{eV}$
FIG. 1. (Color online) The ground state and higher energy isomers of $\mathrm{Mn} @ \mathrm{Sn}_{12}$ cluster. The energies are relative to the ground state $I_{h}$ cage. The symmetry and spin multiplicities are also shown.
Our theoretical results show that the icosahedral $\mathrm{Sn}_{12}$ cage, with the $\mathrm{Mn}$ atom trapped inside the center of the cage is the most preferred structure for $\mathrm{Mn}$-doped $\mathrm{Sn}_{12}$ cluster (Fig. 1). Even though, the $\mathrm{Mn} @ \mathrm{Sn}_{12}$ cluster is an open-shell system, the Jahn-Teller effects are found to be minimal leading to a very small structural distortion of the icosahedral cage. We have examined several other isomers (Fig. 1) and all of them are found to be higher in energy than the endohedral- $I_{h}$ cage structure. The Mn-Sn bond length in the $\mathrm{Mn} @ \mathrm{Sn}_{12}$ cage is calculated to be $3.05 \AA$. Our bond lengths are in good agreement with the previously reported theoretical study ${ }^{7}$ on $\mathrm{Mn} @ \mathrm{Sn}_{12}$ cluster. In order to understand the charge state of Mn inside the cage cluster, one usually analyzes the Mulliken charge distributions. However, it is well known that Mulliken charges can only provide a qualitative picture of the charge distribution, but cannot give accurate quantitative information. To understand the charge state of $\mathrm{Mn}$ and the $\mathrm{Sn}_{12}$ cage cluster, we take a different approach by comparing the equilibrium geometries and $\mathrm{Sn}-\mathrm{Sn}$ bond lengths of $\mathrm{Mn}$ doped stannaspherene with that of $\mathrm{Sn}_{12}^{2-}$. In $\mathrm{Mn} @ \mathrm{Sn}_{12} I_{h}$ cage, the $\mathrm{Sn}-\mathrm{Sn}$ bond length is $3.20 \AA$ which is nearly identical with the previously reported ${ }^{11} \mathrm{Sn}-\mathrm{Sn}$ bond length $(3.19 \AA)$ of the empty $\mathrm{Sn}_{12}^{2-} I_{h}$-cage cluster. The absence of significant structural distortions in the $I_{h}$ cage due to $\mathrm{Mn}$ encapsulation along with the identical $\mathrm{Sn}-\mathrm{Sn}$ bond lengths in $\mathrm{Mn} @ \mathrm{Sn}_{12}$ and $\mathrm{Sn}_{12}^{2-}$ cage indicate that in the case of $\mathrm{Mn} @ \mathrm{Sn}_{12}$ cluster, the tin cage behaves like $\mathrm{Sn}_{12}^{2-}$, and consequently $\mathrm{Mn}$ would remain in a 2+ valence state. These observations are consistent with the previously reported study ${ }^{12}$ on the metal doped stannaspherenes, where $M @ \mathrm{Sn}_{12}$ cage clusters were shown to exist as $M^{2+}$ and $\mathrm{Sn}_{12}^{2-}$. An unusually large highest orbital molecular orbital (HOMO)lowest unocoupled molecular orbital (LUMO) gap of $1.12 \mathrm{eV}$ is observed in the $\mathrm{Mn} @ \mathrm{Sn}_{12}$ cluster. This further highlights the stability of the neutral cage structure. The $\mathrm{Mn} @ \mathrm{Sn}_{12} I_{h}$ cage exhibits a high spin multiplicity of sextet $(2 S+1=6)$ yielding a magnetic moment of $5 \mu_{B}$. Of this, the spin-magnetic moment on the $\mathrm{Mn}$ atom is found to be $4.15 \mu_{B}$ and originates from the $3 d$ states of $\mathrm{Mn}$. The large HOMO-LUMO gap and the high magnetic state of $\mathrm{Mn} @ \mathrm{Sn}_{12}$ observed in the current study are consistent with the previously reported theoretical work. ${ }^{7}$

We then studied the interaction between two $\mathrm{Mn} @ \mathrm{Sn}_{12}$ cage clusters to determine if the endohedral stannaspherenes can retain their cage structures. We have also studied the interaction effects on the magnetic properties of the dimer cage clusters. We examined several structural configurations in which two $\mathrm{Mn} @ \mathrm{Sn}_{12}$ cage clusters were allowed to interact in various orientations with respect to each other (Fig. S1). ${ }^{23}$ We found that the two $\mathrm{Mn} @ \mathrm{Sn}_{12} I_{h}$-cage clusters do maintain their structural integrity, and are bonded at the triangular faces of the icosahedra, with the triangles facing upside down with respect to each other [Fig. 2(a)]. The two
$\mathrm{Mn} @ \mathrm{Sn}_{12} I_{h}$ cages remained nearly intact with only a small distortion in the icosahedron skeletons, resulting in $C_{2 h}$ symmetry for the ground state configuration of the dimer. The most interesting feature of the $\mathrm{Mn} @ \mathrm{Sn}_{12}$ dimer is its spin state. In the ground state, the Mn atoms are antiferromagnetically (AFM) coupled carrying a spin magnetic moment of $+4.04 \mu_{B}$ and $-4.04 \mu_{B}$, respectively. However, a high spin state $(2 S+1=11)$ in which the $\mathrm{Mn}$ atoms are ferromagnetically (FM) coupled is only $0.11 \mathrm{eV}$ higher in energy. In this FM state, the total magnetic moment is $10 \mu_{B}$ with the spin magnetic moment on each $\mathrm{Mn}$ atom being $4.14 \mu_{B}$. The $\mathrm{Sn}-\mathrm{Sn}$ bond length between the two cages is found to be $3.26 \AA$ for AFM state, while it is $3.40 \AA$ for the high spin FM state. Interestingly, the large HOMO-LUMO gap
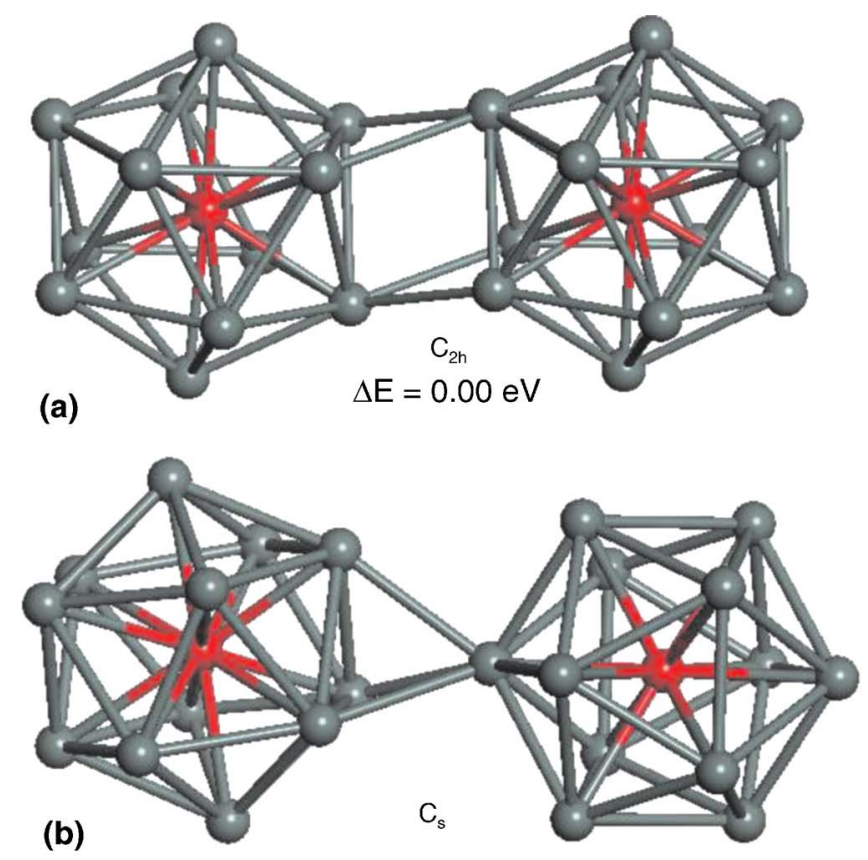

$\Delta \mathrm{E}=0.34 \mathrm{eV}$

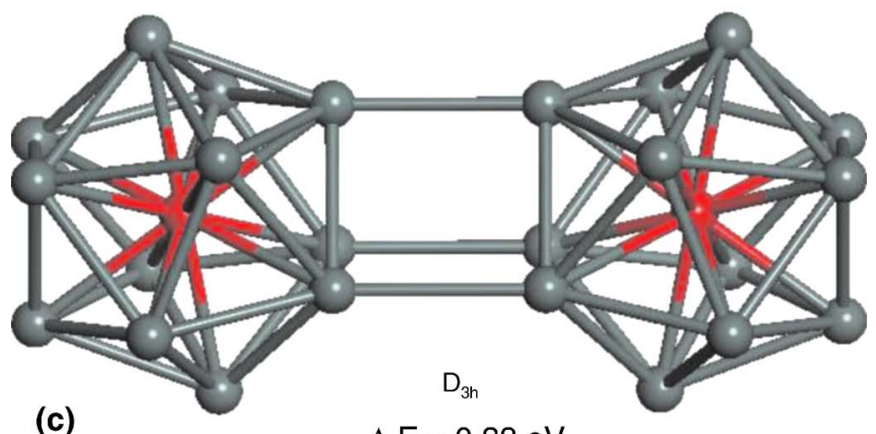

$\Delta E=0.38 \mathrm{eV}$

FIG. 2. (Color online) The optimized structures of $\mathrm{Mn} @ \mathrm{Sn}_{12}$ dimer. (a) represents the lowest energy structure; (b) and (c) represent the higher energy isomers. The relative energy $\Delta E$ of the isomers is also shown. 
$(1.12 \mathrm{eV})$ seen in $\mathrm{Mn} @ \mathrm{Sn}_{12}$ has decreased only slightly due to the dimerization, with the HOMO-LUMO gap of the dimer being $0.88 \mathrm{eV}$. The binding energy of the ground state dimer is $0.73 \mathrm{eV}$, which is significantly larger than that between two $\mathrm{C}_{60}$ fullerenes. There exists a higher energy configuration $(\Delta E=0.34 \mathrm{eV})$ with $C_{s}$ symmetry [Fig. 2(b)], in which the $C_{5}$ axis of one of the $\mathrm{Mn} @ \mathrm{Sn}_{12}$ cage is rotated by 90 with respect to the other cage. In this $C_{s}$ configuration, the $\mathrm{Mn}$ atoms prefer ferromagnetic coupling $\left(4.15 \mu_{B}, 4.11 \mu_{B}\right)$, the total spin of the dimer being $10 \mu_{B}$. However, an AFM state $\left(4.13 \mu_{B},-4.09 \mu_{B}\right)$ is found to be energetically degenerate $(\Delta \varepsilon=0.01 \mathrm{eV})$ with the FM state.

Another structural configuration [Fig. 2(c)], where the two Mn doped stannaspherene cages are joined at the triangular faces is $0.38 \mathrm{eV}$ higher in energy. The spin state for this $D_{3 h}$ symmetric configuration is found to be AFM (singlet state) with $+4.02 \mu_{B}$ and $-4.02 \mu_{B}$ spin moment on the Mn atoms. It is to be noted there that this higher energy configuration results from a $90^{\circ}$ rotation along the $C_{3}$ axis of one of the cages in ground state configuration. Thus, the stability and magnetic nature of the $\mathrm{Mn} @ \mathrm{Sn}_{12}$ dimer are strongly dependent on orientation of the cages with respect to each other. The variations in the stability of $\mathrm{Mn} @ \mathrm{Sn}_{12}$ dimer cluster with the rotation of one $\mathrm{Mn} @ \mathrm{Sn}_{12}$ cage with respect to the other cage is reminiscent of the previously reported ${ }^{24}$ orientation dependent interaction between two $\mathrm{C}_{60}$ molecules.

We have further tested the thermal stability of the ground state $\mathrm{Mn} @ \mathrm{Sn}_{12}$ dimer by carrying out constant temperature molecular dynamics simulations for 1 ps at $300 \mathrm{~K}$. After $1 \mathrm{ps}$, it was found that the dimer cage cluster did not collapse and the monomer remains the cage structure skeleton with a minor distortion, indicating the stability of the magnetic dimer cages at room temperature.

Very recently, Sun et al. ${ }^{25}$ synthesized $\mathrm{Pd}_{2} @ \mathrm{Sn}_{18}^{4-}$ cluster by fusing two endohedral $\mathrm{Pd} @ \mathrm{Sn}_{12}^{2-}$ cages along their $C_{3}$ axis and by removing $\mathrm{Sn}_{3}$ triangular face from each cage unit. In our study, we did not see the fusion of the $\mathrm{Mn} @ \mathrm{Sn}_{12}$ $I_{h}$ cages to occur. Interestingly, when two $\mathrm{Mn} @ \mathrm{Sn}_{12}$ octahedral $\left(O_{h}\right)$ cages were allowed to interact, they coalesced to form $\mathrm{Mn}_{2} @ \mathrm{Sn}_{24}$ cage structure. However, this fused cage cluster was found to be $2.82 \mathrm{eV}$ higher in energy than the ground state $C_{2 h}$ configuration. It is to be noted here that the $\mathrm{Mn} @ \mathrm{Sn}_{12} O_{h}$ cage (Fig. 1) itself is a higher energy isomer $(\Delta E=2.22 \mathrm{eV})$ of $\mathrm{Mn} @ \mathrm{Sn}_{12}$.

Following the completion of this work and during the review process of this manuscript, we learnt of another theoretical study ${ }^{26}$ by Matxain et al. focusing on the magnetic nature of $\mathrm{Mn} @ \mathrm{Sn}_{12}$ dimer cages. Our observations regarding the charge state of $\mathrm{Mn}$ in $\mathrm{Mn} @ \mathrm{Sn}_{12}$ are in agreement with this recently reported study. The authors reported three stable $\left[\mathrm{Mn} @ \mathrm{Sn}_{12}\right]_{2}$ isomers, which are termed as $F-\left[\mathrm{Mn} @ \mathrm{Sn}_{12}\right]_{2}$, $V$-[Mn @ $\left.\mathrm{Sn}_{12}\right]_{2}$, and $E$-[Mn @ $\left.\mathrm{Sn}_{12}\right]_{2}$, with $D_{3 h}, D_{2 h}$, and $D_{5 d}$ symmetry, respectively. However, our calculations reveal that these three isomers are $0.38 \mathrm{eV}, 0.46 \mathrm{eV}$, and $0.64 \mathrm{eV}$ higher in energy, in the same order, than the $C_{2 h}$ symmetric structure reported here (See Fig. S1). ${ }^{23}$

In summary, we have shown that $\mathrm{Mn} @ \mathrm{Sn}_{12}$ not only forms a stable magnetic $I_{h}$-cage cluster but also maintains its structural integrity with minimal structural modification during the interaction with another $\mathrm{Mn} @ \mathrm{Sn}_{12}$ cage even at finite temperature. The magnetic state of the $\mathrm{Mn} @ \mathrm{Sn}_{12}$ dimer depends strongly on the mutual orientation of the two cages indicating that one can change the magnetic coupling between two stannaspherenes by simply rotating one with respect to the other. The high magnetic moment of $\mathrm{Mn} @ \mathrm{Sn}_{12}$, its robust cage structure, stability at room temperature, and its ability to retain its structure when assembled may give raise to some exciting possibilities of designing magnetic nanostructures with the $\mathrm{Mn} @ \mathrm{Sn}_{12}$ cages as building blocks.

This work is supported by the U.S. Department of Energy.

${ }^{1}$ H. W. Kroto, J. R. Heath, S. C. O'Brien, R. F. Curl, and R. E. Smalley, Nature (London) 318, 162 (1985).

${ }^{2}$ W. Kratschmer, L. D. Lamb, K. Fostiropoulos, and D. R. Huffman, Nature (London) 347, 354 (1990).

${ }^{3}$ H. Hiura, T. Miyazaki, and T. Kanayama, Phys. Rev. Lett. 86, 1733 (2001).

${ }^{4}$ S. N. Khanna, B. K. Rao, and P. Jena, Phys. Rev. Lett. 89, 016803 (2002).

${ }^{5}$ V. Kumar and Y. Kawazoe, Phys. Rev. Lett. 88, 235504 (2002).

${ }^{6}$ V. Kumar and Y. Kawazoe, Appl. Phys. Lett. 80, 859 (2002).

${ }^{7}$ V. Kumar and Y. Kawazoe, Appl. Phys. Lett. 83, 2677 (2003), and references therein.

${ }^{8}$ M. Ohara, K. Koyasu, A. Nakajima, and K. Kaya, Chem. Phys. Lett. 317, 490 (2003).

${ }^{9}$ S. N. Khanna and P. Jena, Phys. Rev. Lett. 69, 1664 (1992).

${ }^{10}$ S. Bulusu, X. Li, L. S. Wang, and X. C. Zeng, Proc. Natl. Acad. Sci. U.S.A. 103, 8326 (2006).

${ }^{11}$ L. F. Cui, X. Huang, L. M. Wang, D. Y. Zubarev, A. I. Boldyrev, J. Li, and L. S. Wang, J. Am. Chem. Soc. 128, 8390 (2006).

${ }^{12}$ L. F. Cui, X. Huang, L. M. Wang, J. Li, and L. S. Wang, Angew. Chem., Int. Ed. 46, 742 (2007).

${ }^{13}$ J. P. Perdew and Y. Wang, Phys. Rev. B 45, 13244 (1992).

${ }^{14}$ B. Delley, J. Chem. Phys. 92, 508 (1990); B. Delley, J. Chem. Phys. 113, 7756 (2000).

${ }^{15}$ B. Delley, Phys. Rev. B 66, 155125 (2002).

${ }^{16}$ G. Kresse and J. Hafner, Phys. Rev. B 47, 558 (1993).

${ }^{17}$ G. Kresse and J. Furthmüller, Phys. Rev. B 54, 11169 (1996).

${ }^{18}$ G. Kresse and D. Joubert, Phys. Rev. B 59, 1758 (1999).

${ }^{19}$ P. E. Blöchl, Phys. Rev. B 50, 17953 (1994).

${ }^{20}$ S. Nosé, J. Chem. Phys. 81, 511 (1984).

${ }^{21}$ S. Nosé, Prog. Theor. Phys. Suppl. 103, 1 (1991).

${ }^{22}$ D. M. Bylander and L. Kleinman, Phys. Rev. B 46, 13756 (1992).

${ }^{23}$ See EPAPS Document No. E-APPLAB-92-042811 for the ground state and other higher energy isomers of $\mathrm{Mn} @ \mathrm{Sn}_{12}$ dimer, along with their relative energies and preferred spin states. DOS and PDOS are also given for $\mathrm{Mn} @ \mathrm{Sn}_{12}$ dimer. For more information on EPAPS, see http:// www.aip.org/pubservs/epaps.html.

${ }^{24}$ F. Tournus, J. C. Charlier, and P. Melinon, J. Chem. Phys. 122, 094315 (2005).

${ }^{25}$ Z. M. Sun, H. Xiao, J. Li, and L. S. Wang, J. Am. Chem. Soc. 129, 9560 (2007).

${ }^{26}$ J. M. Matxain, M. Piris, E. Formoso, J. M. Mercero, X. Lopez, and J. M. Ugalde, ChemPhysChem 8, 2096 (2007). 